\title{
Cesarean scar pregnancy: clinicians challenge
}

\section{Priyanka Harshavardhan Vora ${ }^{1 *}$, Vandana Bansal ${ }^{2}$}

\begin{abstract}
${ }^{1}$ Department of IVF, ${ }^{2}$ Department of Obstetrics and Gynecology, Nowrosjee Wadia Maternity Hospital, Parel, Mumbai
\end{abstract} 400014, Maharashtra, India

Received: 10 February 2017

Revised: 19 March 2017

Accepted: 23 March 2017

\section{*Correspondence: \\ Dr. Priyanka Harshavardhan Vora, \\ E-mail: priyav865@gmail.com}

Copyright: () the author(s), publisher and licensee Medip Academy. This is an open-access article distributed under the terms of the Creative Commons Attribution Non-Commercial License, which permits unrestricted non-commercial use, distribution, and reproduction in any medium, provided the original work is properly cited.

\section{ABSTRACT}

A case of caesarean scar ectopic pregnancy managed diagnosed early on transvaginal ultrasound and managed successfully by sequential approach of methotrexate and hysteroscopic removal preserving the woman's fertility is discussed below. Ectopic pregnancy i.e. implantation of the blastocyst outside the endometrium of the uterine cavity occurs in $1.9 \%$ of reported pregnancies.

Keywords: Cesarean ectopic hysteroscopy, Pregnancy

\section{INTRODUCTION}

Ectopic pregnancy i.e. implantation of the blastocyst outside the endometrium of the uterine cavity occurs in $1.9 \%$ of reported pregnancies. ${ }^{1}$ Cesarean scar ectopic is a fairly uncommon presentation wherein the conceptus is implanted into the site of previous caesarean section. However, the increased proportions of caesarean sections worldwide have led to increase of this clinical entity. Various management options from medical management to surgical techniques are available, however due to unavailability of any standardized protocol the approach becomes challenging. Timely diagnosis and stepwise approach to preserve the fertility of a child bearing woman in case of failed medical management but successful conservative approach is discussed below.

\section{CASE REPORT}

A 28-year-old G5P1L1SA1MTP2, presented with amenorrhoea of 5 weeks and a positive pregnancy test. Her first pregnancy was a spontaneous complete abortion of two and a half months. Her second pregnancy was an uneventful emergency lower segment caesarean pregnancy at 9 months due to oligohydramnios with a healthy five-year-old male child. In her third and fourth pregnancies, she underwent a medical termination followed by check curettage at one and a half months each. She was undergoing infertility treatment in view of failure to conceive since two and half years post the last MTP and had undergone two cycles of intrauterine insemination which had failed. The current pregnancy was a spontaneous conception. Transvaginal scan showed a caesarean scar pregnancy of 5 weeks with a gestational sac diameter of $0.2 \mathrm{~cm}$ (Figure 1).

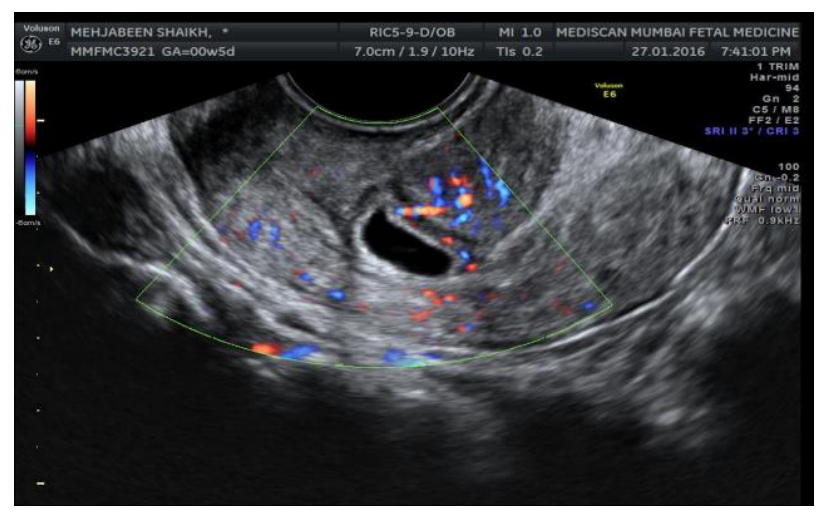

Figure 1: Ultrasound view. 
Yolk sac was seen. No fetal pole or cardiac activity was present. She was in good general condition with a pulse rate of $78 / \mathrm{min}$ and blood pressure of $120 / 70 \mathrm{~mm} \mathrm{Hg}$. Per abdomen was soft with no tenderness, guarding and rigidity. Gynaecological examination was avoided in view of ultrasound suggesting caesarean scar ectopic. Laboratory tests for complete blood count, liver and renal function tests were within normal limits. Serum HCG was $7094 \mathrm{IU} / \mathrm{ml}$. Since the patient wished to preserve her fertility, a conservative approach was implemented. Four doses of methotrexate were given intramuscularly, a dose of $1 \mathrm{mg} / \mathrm{kg}$ alternating with leucovorin rescue of 0.1 $\mathrm{mg} / \mathrm{kg}$. Serial serum HCG values were performed at weekly intervals. Post chemotherapy reading showed an initial increase on day 3 to $9327 \mathrm{IU} / \mathrm{ml}$ followed by weekly decline to 7662 to 3415 to 1247 to 1114 to 116.37 IU/ml. At 11 week's post chemotherapy, the HCG value dropped to $<100 \mathrm{Iu} / \mathrm{ml}$. Follow up ultrasound however showed a persistent gestational sac not reducing significantly in size and patient failed to resume menses. Hysteroscopic guided resection of the sac was performed. The sac seen on hysteroscopy was necrotic in the scar region with no vascularity and minimal flimsy adhesions (Figure 2). The procedure was uneventful with negligible blood loss and the patient was discharged in stable condition one day after surgery.

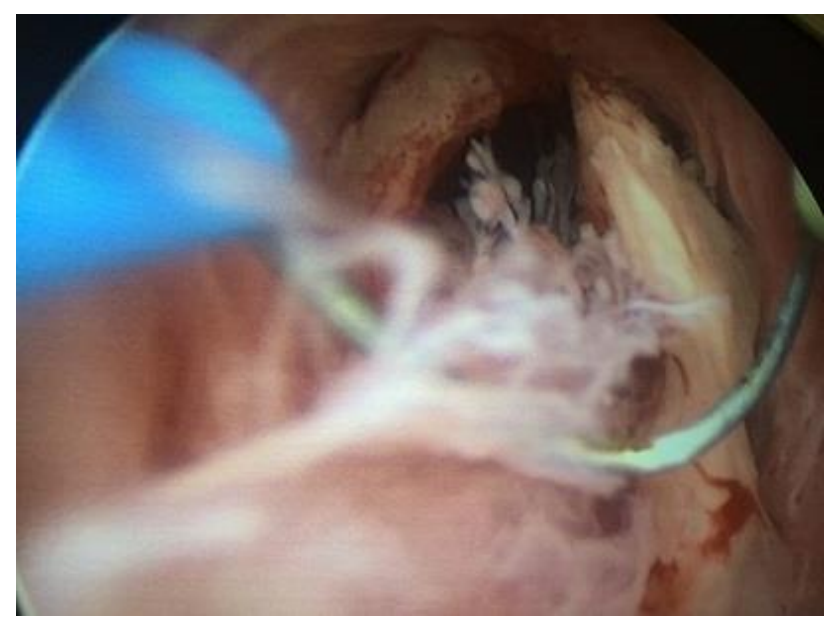

Figure 2: Hysteroscopic resection.

\section{DISCUSSION}

A particular complication of a pregnancy after caesarean delivery $(\mathrm{CD})$ is the implantation of the gestational sac in the hysterotomy scar, known as a "cesarean scar pregnancy" (CSP). This condition is referred as "caesarean ectopic pregnancy" or simply "cesarean scar ectopic". The incidence of CSP has been estimated to range from $1 / 1800-1 / 2216$ and it constitutes $6.1 \%$ of all ectopic pregnancies in women with history of caesarean deliveries. The pathophysiology is the invasion of the blastocyst in the myometrium through minimal communication between the previous cesarean scar and the endometrial cavity. Risk factors include number of prior cesarean sections, dilatations and curettage, hysterotomy, myomectomy, abnormal placentation, manual removal of placenta, short time interval between the cesarean delivery and the current pregnancy and retroversion of the uterus which may lead to greater cesarean scar dehiscence, increasing the chance of implantation of the gestational sac in this region. ${ }^{1,2}$ The patient may manifest as being asymptomatic to vaginal bleeding with or without abdominal pain. In severe cases patient may present with massive bleeding, hemoperitoneum, shock and even death. ${ }^{2,3}$

In the presence of a positive pregnancy test, a CSP is diagnosed by transvaginal ultrasound using the following criteria;

- Visualization of an empty uterine cavity as well as an empty endocervical canal

- Detection of the placenta and/or a gestational sac embedded in the hysterotomy scar

- In early gestations ( $\leq 8$ weeks), a triangular gestational sac that fills the niche of the scar and at $\geq 8$ postmenstrual weeks this shape may become rounded or even oval

- A thin (1-3 mm) or absent myometrial layer between the gestational sac and the bladder

- A closed and empty cervical canal

- The presence of embryonic/fetal pole and/or yolk sac with or without heart activity

- The presence of a prominent and at times rich vascular pattern at or in the area of a CD scar.

According to the classification of CSP by Vial et al, there are two different types of CSP. The first type (CSP-I) is caused by implantation of the amniotic sac into the previous CS with progression of pregnancy toward the cervico-isthmic space and the uterine cavity. Such a situation may allow a viable birth, but at an increased risk of massive bleeding from the site of implantation. The second type (CSP-II) is caused by deep implantation into a CS defect with infiltrating growth into the uterine myometrium and bulging from the uterine serosal surface of the uterus. The thickness of uterine myometrium between the sac and the bladder wall is usually $<4 \mathrm{~mm}$. Because of the high risk of uterine rupture with lifethreatening hemorrhage during the first trimester, CSP-II may result in emergency hysterectomy. Once CSP-II is diagnosed, termination of the pregnancy should be considered. Thus, timely management with early and accurate diagnosis that allows the successful preservation of the uterus is very important. ${ }^{4}$ MRI can be performed when diagnosis by transvaginal Colour Doppler is difficult. The differential diagnosis includes an abortion in progress, molar pregnancy and cervical ectopic pregnancy. The treatment options depend on the patient's hemodynamic condition, severity of vaginal bleeding, gestational age, $\beta \mathrm{hCG}$ levels, demonstration of fetal cardiac activity, need for future childbearing, and the available infrastructure and expertise. Non-surgical treatment may be expectant, medicated with systemic or local methotrexate. This is done when $\beta$ hCG levels are 
less than $5000 \mathrm{mIU} / \mathrm{ml}$ and myometrial thickness is less than $2 \mathrm{~mm}$ with a success rate of $71-80 \%$ with only $6 \%$ requiring hysterectomy. ${ }^{2}$ If the patient presents late with a uterine rupture or major bleeding, surgery is unavoidable. Surgical treatment may be via uterine curettage, hysteroscopic removal, uterine artery embolization or hysterectomy. In cases of intractable haemorrhage intrauterine balloon tamponade with foleys catheter, local vasopressin injection, shirodkars suture and bilateral uterine artery ligation. ${ }^{2}$ Hysteroscopic resection was used to treat CSP-I, but not CSP-II. ${ }^{4}$ Laproscopy is mainly done for a deeply implanted pregnancy growing towards the bladder. It involves scar pregnancy excision, vasopressin injection to minimised bleeding, bipolar diathermy to minimised bleeding and uterine defect closure with endoscopic suturing. Exploratory laparotomy must be sorted to in impending or confirmed cases of uterine rupture. ${ }^{2}$ In women who undergo UAE, local administration of methotrexate is suggested to be a better route compared to its systemic administration because of the theoretical reduced efficacy of methotrexate resulting from decreased blood supply to the pregnancy ${ }^{3}$. During the outpatient follow-up ultrasound, be repeated when there are new episodes of bleeding and $\beta$-hCG reaches a negative value or after 3 months of drug treatment. ${ }^{1}$

\section{CONCLUSION}

The early diagnosis of caesarean scar ectopic pregnancy via transvaginal ultrasound is fundamental for a successful conservative treatment. A sequential combined approach of systemic methotrexate to reduce the vascularity followed by hysteroscopic excision reduces morbidity and risk of torrential haemorrhage.

Funding: No funding sources

Conflict of interest: None declared

Ethical approval: Not required

\section{REFERENCES}

1. de Freitas Leite J, Fraietta R, Júnior JE. Local management with methotrexate of cesarean scar ectopic pregnancy with live embryo guided by transvaginal ultrasound: a case report. J Rev Assoc Med Bras. 2016;62(2).

2. Patel MA. Scar ectopic pregnancy. J of Obstet Gynaecol India. 2015;65(6):372-5.

3. McLaren R, McCalla S, Irani M. Conservative management of cesarean scar ectopic pregnancy with fetal heart activity and a very high $\beta$-hCG. J of Rep Obstet Gynecol. 2015;1-3.

4. Wang HY, Zhang J, Li YN, Wei W, Zhang DW, Lu YQ, et al. Laparoscopic management or laparoscopy combined with transvaginal management of type II cesarean scar pregnancy. J Soci Laproendoscop Surg. 2013;17(2):263-72.

Cite this article as: Vora PH, Bansal V. Cesarean scar pregnancy: clinicians challenge. Int J Reprod Contracept Obstet Gynecol 2017;6:2101-3. 